\title{
Sunshine exposure assessment and vitamin D status in healthy adults
}

\author{
T. R. Hill ${ }^{1}$, A. J. Lucey ${ }^{1}$, G. Dawson ${ }^{1}$, J. M. Wallace ${ }^{4}$, G. Horigan ${ }^{4}$, M. S. Barnes ${ }^{4}$, \\ M. P. Bonham ${ }^{4}$, N. Taylor ${ }_{1}^{1}$, E. M. Duffy ${ }^{4}$, K. Seamans $^{1}$, S. Muldowney ${ }_{1}^{1}$, A. P. Fitzgerald ${ }^{3}$, \\ A. Flynn ${ }^{1}$, J. J. Strain ${ }^{4}$, M. Kiely ${ }^{1}$ and K. D. Cashman ${ }^{1,2}$ \\ ${ }^{1}$ Departments of Food and Nutritional Sciences, ${ }^{2}$ Medicine and ${ }^{3}$ Epidemiology and Public Health, \\ University College Cork, Cork, Republic of Ireland and ${ }^{4}$ Northern Ireland Centre for Food and Health, University of Ulster, \\ Coleraine BT52 1SA, UK
}

Sun exposure is the major source of vitamin D for human subjects ${ }^{(1)}$. Investigation of the underlying reasons for low vitamin D status as well as establishment of appropriate dietary recommendations for vitamin D would benefit from data on the contribution of sunshine exposure to vitamin D status. The objective of the present study was to determine whether direct and indirect UV sunlight assessment tools (polysulphone dosimeters and sunshine-exposure questionnaires respectively) are capable of predicting late-summer vitamin D status among adults in Ireland. Fasting serum 25-hydroxyvitamin D (S-25(OH)D) concentrations were measured by ELISA in 232 adults aged $20-40$ years (113 from Cork city and 119 from Coleraine) during October 2006. Habitual sun exposure and habitual outdoor activity from summer 2006 were assessed using a recently-developed sunshine-exposure questionnaire. Polysulphone film badges, used to measure personal exposure to UV radiation $^{(2)}$ were worn by subjects to record cumulative sun exposure over a 6 d period during July and August 2006. During the same period subjects logged personal daily habits and weather in sun-exposure diaries. Multiple linear regression was used to investigate the association between sun-exposure variables and $\mathrm{S}-25(\mathrm{OH}) \mathrm{D}$ concentrations.

Of a total of thirty-seven questions on the sun-exposure questionnaire, sun preference ('During the sunny months would you; avoid, sometimes stay or enjoy staying in direct sunshine?') and estimated period (mind) spent outdoors were positive predictors of late-summer $\mathrm{S}-25(\mathrm{OH}) \mathrm{D}$ levels. Using multiple linear regression for both sunshine assessment variables separately and after adjustment for potential confounders, avoidance of the sun had a strong negative association, while period spent outdoors had a positive association with $\mathrm{S}-25(\mathrm{OH}) \mathrm{D}$ levels. There was a weakly positive correlation between $\mathrm{S}-25(\mathrm{OH}) \mathrm{D}$ and total standard erythemal dose $(\mathrm{SED} ; P=0.06)$ as measured by polysulphone film badges. This trend remained after adjustment for potential confounders $(P=0.057)$.

\begin{tabular}{lccc}
\hline Sunshine exposure variable*..... & $\beta$ & SE & $95 \%$ CI \\
\hline Sun preference (prefer staying in sun, & & & $P$ \\
$\quad$ reference group) & & & $-0.553,-0.239$ \\
$\quad$ Avoid sun & -0.330 & 0.08 & $-0.331,-0.118$ \\
$\quad$ Sometimes stay in sun & -0.278 & 0.054 & $0.000,0.002$ \\
Total period (min/d) spent outdoor & 0.175 & 0.000 & $0.000,0.014$ \\
$\quad$ during sunny months & 0.146 & 0.004 & 0.001 \\
Total SED & & 0.057 \\
Multiple linear regression with S-25(OH)D as dependent variable. *Three separate regression models were constructed (one for each sunshine \\
$\quad$ assessment variable) and each model included centre, age, vitamin D and Ca intake and BMI as confounders.
\end{tabular}

Selected questions from the sun-exposure questionnaire relating to sun preference and estimated period spent outdoors, could be used to stratify participants into low and high S-25(OH)D. Use of polysulphone film badges, which were significantly but weakly associated with $\mathrm{S}-25(\mathrm{OH}) \mathrm{D}$, was a less promising approach.

This work was supported by the UK Food Standards Agency.

1. Department of Health (1998) Nutrition and Bone Health with Particular Reference to Calcium and Vitamin D. London: The Stationery Office.

2. Diffey B (1984) Photodermatology 1, 151-157. 